\title{
A Registry BASED Discovery MECHANISM FOR E-LEARNING WEB SERVICES
}

\author{
Demian Antony D’Mello ${ }^{1}$, Pramod Prabhu², Naveen Baliga ${ }^{3}$, Nikhil \\ Misquith $^{4}$ \\ and Flexon Fernandes ${ }^{5}$ \\ Department of Computer Science and Engineering, St. Joseph Engineering \\ College, Mangalore, INDIA - 575028 \\ ${ }^{1}$ demian.antony@gmail.com, 2 pramodprabhu123@gmail.com, \\ ${ }^{3}$ naveenbaliga007@yahoo.com, ${ }^{4}$ aaronmisquith@live.com, \\ ${ }^{5}$ flexonfer@gmail.com
}

\begin{abstract}
E-learning is currently taking the shape of a Web Service in various applications i.e. learners can search for suitable content, book it, pay for it and consume it. This paper shows how the search aspects for e-learning content can technically be combined with the recent standardization efforts that aim at content exchangeability and efficient reuse. A repository for learning object publication and search is proposed that essentially adapts the UDDI framework used in commercial Web Services to the e-learning context. To adopt Web Services technology towards the reusability and aggregation of e-learning services, the conceptual Web Services architecture and its building blocks need to be augmented. The objective of this research is to design broker based registry architecture for $e$ - Web services which facilitates effective elearning content/service discovery for the consumption or composition. The implementation followed by experimentation showed that, the proposed e-learning discovery architecture facilitates effective discovery with moderate performance in terms of overall response.
\end{abstract}

\section{KEYWORDS}

Web Service, E-Learning, E-Learning Discovery, E-Learning Broker \& E-Learning Registry

\section{INTRODUCTION}

With the development of Internet and Web, information can be shared by web sites around the world [1]. Knowledge can be assimilated as well as disseminated across the boundaries of learning organizations. The objective of any Internet based system is to facilitate information sharing. E-learning is a learning mode of technology enhanced learning based on Web technology.

\subsection{E-Learning}

E-learning is a fast growth application in the world, it is widely used in universities, enterprises, communities, and over one million users now are learning through it [2]. As a typical technicaldriven application, the development of e-learning has close relationship with IT technologies such as computer network, multimedia technology and etc. The mainstream of e-learning systems now are mostly server-centric based, users access the same e-learning servers to get served (view courseware, have some quiz, discuss etc), and the user-interfaces are mainly web based for

Sundarapandian et al. (Eds): CoNeCo,WiMo, NLP, CRYPSIS, ICAIT, ICDIP, ITCSE, CS \& IT 07, pp. 431-443, 2012. (C) CS \& IT-CSCP 2012

DOI : $10.5121 /$ csit.2012.2442 
convenience. That kind of e-learning system runs well while the number of users is not so large, and the content user access is mainly Web page based.

\subsection{Basic Infrastructure for Web Services}

The term Web Services describes a standardized way of integrating Web-based applications using the XML, Simple Object Access Protocol (SOAP), WSDL and UDDI open standards over an Internet protocol backbone [3]. XML is used to tag the data, SOAP is used to transfer the data, WSDL is used for describing the services available and UDDI is used for listing what services are available [4]. These facilities allow the integration of systems written in different languages and running on computers with different platforms. A Web Service is essentially a stand-alone software component that has a unique URI (the Uniform Resource Identifier is a unique address) and that operates over the Internet and particularly the Web. The basic premise is that Web Services have a provider and (hopefully) users or subscribers. Web Services can be combined to build new ones with a more comprehensive functionality [5]. Clearly, Web Services need to be interoperable. Moreover, they have to be independent of the operating systems. They should work on every Web Service engine regardless of their programming language. To achieve these goals, Web Services are commonly based on standards. Currently, the most common ones are the XMLbased specifications SOAP, UDDI, and WSDL.

\subsection{Early E-Learning Initiatives}

The main players in the e-learning systems are the learners and the authors; others include trainers and administrators [6]. Authors (which can also be teachers or instructional designers) create content, which is stored under the control of a LMS and typically in a database. Existing content can be updated and also reused in other e-learning systems. The administrator controls the LMS [7]. The core of an e-learning system, which is under the control of an administrator, typically consists of a LMS or LCMS. The LMS interacts with a run-time environment, which is addressed by learners, who in turn may be coached by a trainer. The interesting aspect of this idea is the fact that these three components of an e-learning system can be logically and physically distributed, i.e., installed on distinct machines and offered by different providers or content suppliers. In order to make such a distribution feasible, standards such as IMS and SCORM ensure plug-and-play compatibility to a large extend.

E-learning systems often do not address only a special kind of learner; moreover, they may be implemented in such a way that a customization of features and the content appearance is adapted to the needs of an individual learner [8]. Learners vary in pre-knowledge, abilities, goals for approaching a learning system, pace of learning, way of learning, and the time (and money) they are able to spend on learning. Thus, the target group of learners is very heterogeneous; ideally, a system is able to provide and present content for every group or some of them, in order to be suitable, for example, for a student who wants to learn about database concepts or for a company employee who wants to become familiar with company-internal processes and their execution. To fulfil the needs of such a flexible system, a learning platform has to meet a number of requirements, including the integration of a variety of materials, the potential deviation from predetermined sequences of actions, personalization and adaptation, the verifiability of work and accomplishments [9].

Content consumed by learners and created by authors is commonly handled, stored, and exchanged in units of learning objects (LOs) [10]. Basically, LOs are units of study, exercise, or practice that can be consumed in a single session, and they represent reusable granules that can be created no matter what kind of delivery medium is used [11]. The LOs can be accessed dynamically, e.g. over the Web. Ideally, LOs can be reused by different LMS and plugged together to build classes that are intended to serve a particular purpose or goal [12]. Accordingly, 
LOs need to be context-free, which means that they have to carry useful description information on the type and context in which they may be used.

\subsection{Challenges in E-Learning System Development}

In literature researchers have proposed a few mechanisms to solve the issues related to e-learning service discovery and architectures [13, 14]. With the rapid development of e-learning services and applications over the Web, it appears some new requirements:

- The number of users increases fast, the load of the e-learning server makes the system slower and the risk of crash increases. E-learning system needs a new method to balance the load.

- Users are more scattered and so is the content. We need to find content which is suitable for the users and also reduce the effort of the users to find content for the topic of their interest.

- The e-learning users will ask for more vivid courseware in e-learning including broader range of multimedia content, including audio, video, film, as well as text, image and picture and more.

Hence for these reasons, we need to find effective solutions to integrate various parts of elearning into a single Web Service where the user may find all his interests in a single place. Often there are several e-learning services which go unnoticed or are popular only in the specific part of the world and the rest of them have no clue about the services offered by them. This makes it difficult for user to search for his contents and hence spend more time in searching for the topic than actually learning. Also the user has to remember or finds the difficulty to navigate to all the e-learning service providers. Hence the user finds it very difficult to compare the quality of the content because the rating of the content is only local to that content provider and not with respect to all the providers. Also the content should also be of the standard of the type of user. There are also instances when the user has to stop the current activity of learning and when the user resumes learning he has to again go through all the steps in accessing all the information so that he can just resume from where he had last stopped. This makes the user feel that there should be a centralized architecture for e-learning so that he can avail all these services at one portal, which can remember all the preferences and settings of the user so that it can simplify the work of searching, categorizing and presentation of information.

A service-oriented e-learning system results from a perception of the various tasks and activities that are contained in such a system as processes or as workflows; using appropriate encodings of objects and tasks in UDDI and WSDL forms and documents enable broad exchanges, flexible compositions, and highly customized adaptations possible [15]. This even allows a reuse of services already offered on the Web, such as multimedia streaming, chat rooms, or conferencing etc. By combining several e-learning services; we reduce the effort of the user for searching for his learning materials, and can spend more time in learning of the topic of his interest. Thus by providing an effective platform and simplifying architecture of e-learning we can make more users who tend to stay away from e-learning because of the complexities involved adopt this type of approach to learning.

The organization of this paper is as follows. Section 2 describes the e-learning service discovery problem with an illustration. In section 3, the authors propose architecture for the e-learning services which facilitates publishing and discovery. The e-learning service discovery mechanism is explored in section-4. Section 5 explains the implementation and experiment details. Section 6 draws conclusions. 


\section{E-LEARNing SERVICE Discovery PROBLEM}

In the world of Web and Web Services, reusability and interaction between Web Services is an interesting issue which is open for research. The success of this rising technology is dependent on the effectiveness of techniques used to handle the requester's complex functional and nonfunctional demands on services. To facilitate widespread adoption, there is a need to augment the existing Web Service architecture and mechanisms for the Web Service discovery and compositions.

E-learning systems often do not address only a special kind of learner; moreover, they may be implemented in such a way that a customization of features and the content appearance is adapted to the needs of an individual learner. Learners vary significantly in knowledge, abilities, goals for approaching a learning system, pace of learning, way of learning and the time. Thus, the target group of learners is very heterogeneous; ideally, a system is able to provide and present content for every group or some of them, in order to be suitable. To fulfil the needs of such a flexible system, a learning platform has to meet a number of requirements, including the integration of a variety of materials, the potential deviation from predetermined sequences of actions, personalization and adaptation, and the verifiability of work and accomplishments. With all the above requirements in mind we define the e-learning service discovery as follows:

Problem Definition: Let $\mathrm{SP}=\left\{\mathrm{p}_{1}, \mathrm{p}_{2} \ldots \mathrm{p}_{\mathrm{n}}\right\}$ where $\mathrm{p}_{1}, \mathrm{p}_{2} \ldots \mathrm{p}_{\mathrm{n}}$ are the individual E-Learning service providers. Let $\mathrm{R}=\left\{1_{1}, 1_{2} \ldots 1_{\mathrm{m}}\right\}$ where $1_{1}, 1_{2} \ldots 1_{\mathrm{m}}$ are the individual E-Learning service/content requesters. The problem is to select the best (most suitable) e-learning service/content provided by the registered service provider 'pi' for the service requester.

The e-learning resources are often scattered all over the globe of the e-learning domain. Therefore it becomes very difficult for the learner to search for the required resources, since navigating through all the e-learning services becomes a time consuming and tedious task. Our challenge is to design a broker based architecture which facilitates registering and publishing of e-learning services by the service providers and searching for the appropriate e-learning resources and composing them for the e-learner's benefit. This makes the user feel that there should be a centralized architecture fore-learning so that he can avail all these services at one portal, which can remember all the preferences and settings of the user so that it can simplify the work of searching, categorizing and presentation of information. As an illustration, consider a learner who wants to use the traditional e-learning system available at his disposal to have an in-depth study on a particular subject e.g. Database Management System, then the user must specify his needs in the e-learning tool. In any e-learning tool the learner must specify the subject as well as the type of learning material required for the study, e.g. DBMS online lectures, videos, articles, text books and so on. The e-learning system searches for these resources within its domain and returns the services provided by its service providers. These results are numerous and are scattered all over the internet. There is also a possibility of redundant results being displayed. The learner will have to manually check each of these results to confirm if the result meets his demands or not. This time consuming problem is what we refer to as e-learning discovery. To realize the solution to these problems, the conceptual Broker based architecture needs to be augmented with new architectural roles and operations.

\section{ARCHITECTURE FOR E-LEARNING SERVICE DISCOVERY}

The proposed solution describes the design and implementation of Web Service architecture for e-learning. By moving offline activities online, Web Services enable partners to (re)use easily applications via the Internet.

The proposed methodology includes: 
1. Design of broker for e-learning Web Service discovery

2. Extension to existing WSDL document to incorporate e-Learning service descriptions

3. Design of e-Learning registry to discovery and save e-learning specific descriptions

4. Design and Implementation of e-learning discovery mechanism for the learner's simple learning needs

\subsection{Abstract Architecture E-learning Service Discovery}

Building a non-centralized system by combining several Web Services to achieve the same functionality as in traditional e-learning systems leads to the problems of managing the content for the learners and searching for services to gain the desired functionality in the moment of demand. The requester's often use the UDDI registry to search for Web Services. The UDDI data entities provide support for defining both business and service information which conventionally stored at central site. To enable learner's to access learning services, we need to develop the broker based architecture which facilitates both service centric and learning centric information storage and retrieval. Figure 1 shows the abstract architecture of e-learning Web service architecture for publishing and discovery.

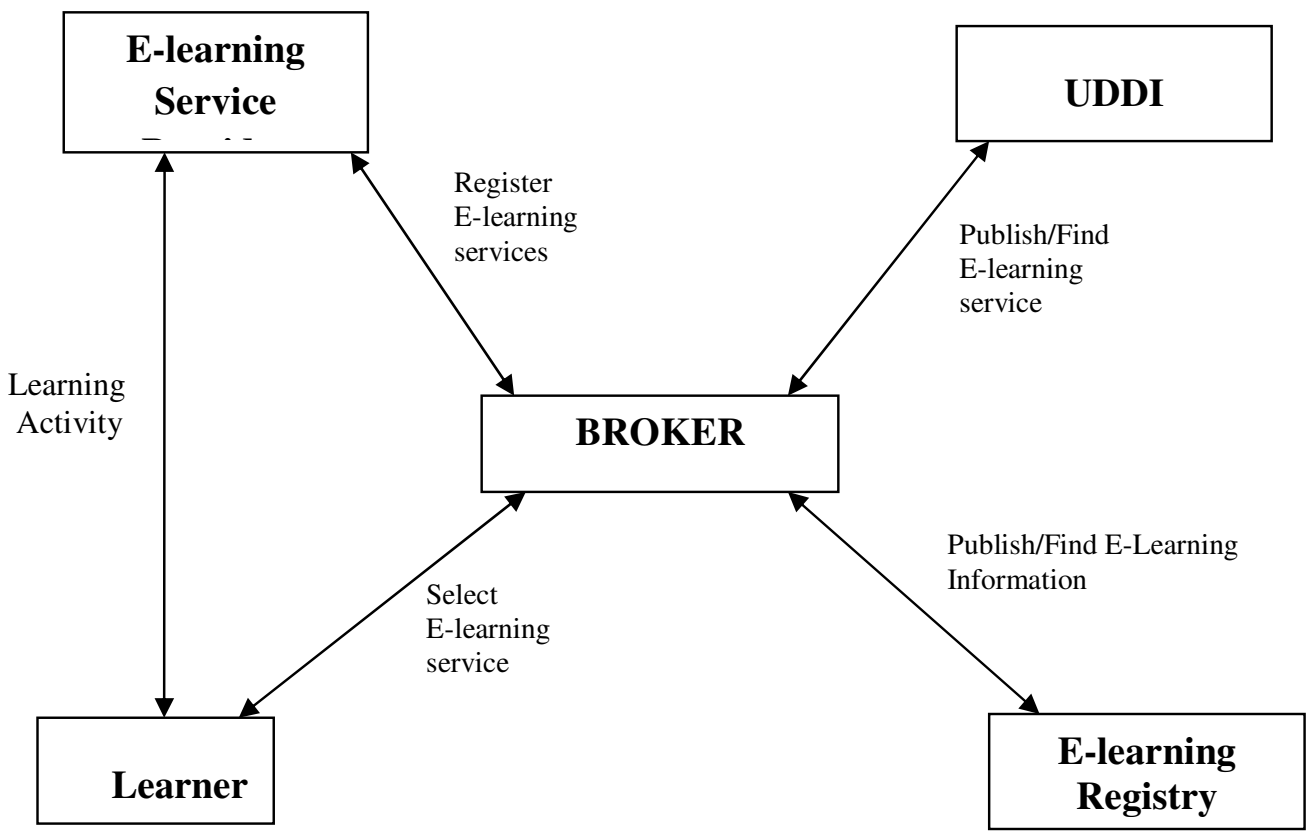

Figure 1. Broker Based Architecture for E-Learning Services

The proposed architecture involves five roles and seven architectural interactions among roles. The roles are: Learner, Provider, Broker, UDDI and E-Learning Registry. The operations/activities among them are: Register E-learning service, Publish Service, Publish eLearning Information, Find Service, Find e-Learning Information, Bind E-Learning Service and Select E-Learning Service.

\subsubsection{Learner/e-learning service requester}

The learner is the one who wants the E-learning service to consume e-content. Learner could be a student, teacher, philosopher or professional. The learner doesn't have a direct access to the elearning registry. The learner should communicate with the broker and search for the required services available in UDD. If the required service is available then the broker gives the URI of the service provider using which the learner can communicate with the service provider. 


\subsubsection{E-learning Service Provider}

The e-learning service provider provides various services to the learners. E-learning service providers can be categorized based on the nature of the service and the type of learning content and the topic of learning. The e-learning service providers must first register its service with the broker in order to be available to the learners.

\subsubsection{UDDI}

UDDI registry is normally used to search and publish Web Services. The UDDI data entities provide support for defining both business and service information. The service description information defined in WSDL is complementary to the information found in a UDDI registry. The UDDI refers to the entity tables defined in it, to provide the services requested by the Broker. The entities referred by the UDDI are Business Entity, Business Service and Binding Template. The service specific information of e-learning services is published in UDDI for the global visibility.

\subsubsection{E-learning Registry}

The E-learning registry is designed to discover and save e-leaning specific descriptions and other necessary information related to learning contents. The UDDI does not provide facilities to store content information. Thus we need to provide an additional registry that can handle such data. This registry can handle e-learning specific information required for the discovery of services by various e-learners.

\subsubsection{E-Learning Broker}

The important role in this e-learning architecture is played by the e-learning Service broker. The broker could be a Web Service or a Portal with service API using which a learner can avail various learning services. The broker communicates with the service provider who wants to register and provide its learning materials. The broker also interacts with the learner when he wants to search for a particular learning material. It also searches the e-learning registry and UDDI when the service provider and the learners perform publish and search operations respectively.

\subsection{Internal Architecture of the E-Learning Broker}

The e-learning broker consists of five components which facilitate the effective e-learning service publishing and discovery. Figure 2 shows the detailed internal architecture of e-learning service broker. The component descriptions are given below:

\subsubsection{Service Selector}

This module helps to select the relevant service from the set of services retrieved from the UDDI. This module is also responsible for the communication with Data Dictionary. The service selector also provides the services for the service composition (if any) and then this service is returned to the learner who requested/ searched for that service.

\subsubsection{Data Dictionary}

It contains domain specific information related to E Learning. Data Dictionary is also called as a metadata repository. It has some information related to the data in the UDDI. The contents of it are automatically updated as changes occur in the database. It may interact with the modules and provide the necessary information for its functioning.

\subsubsection{Service Publisher}

For the provider, this module will helps to register e-learning service with the broker. Service publisher then publishes the service specific information into UDDI and e-learning specific information in the e-learning registry. 


\subsubsection{Request Analyzer}

From the learners' point of view, this module helps the learner to search for e-learning service using this broker. This then, retrieves the information about the service provider from the UDDI and the E-learning information about the content from the E-learning registry.

\subsubsection{E-Learning Registry}

This registry is used to store the e-learning content specific information into registry. The registry contains one record for each service with service Id, learning object Id, learning action, learning object name, learning object type, learning object qualifier and binding template key. The architecture assumes that, the mechanism adopts the functional semantic [16] concepts to build both request query and service publishing.

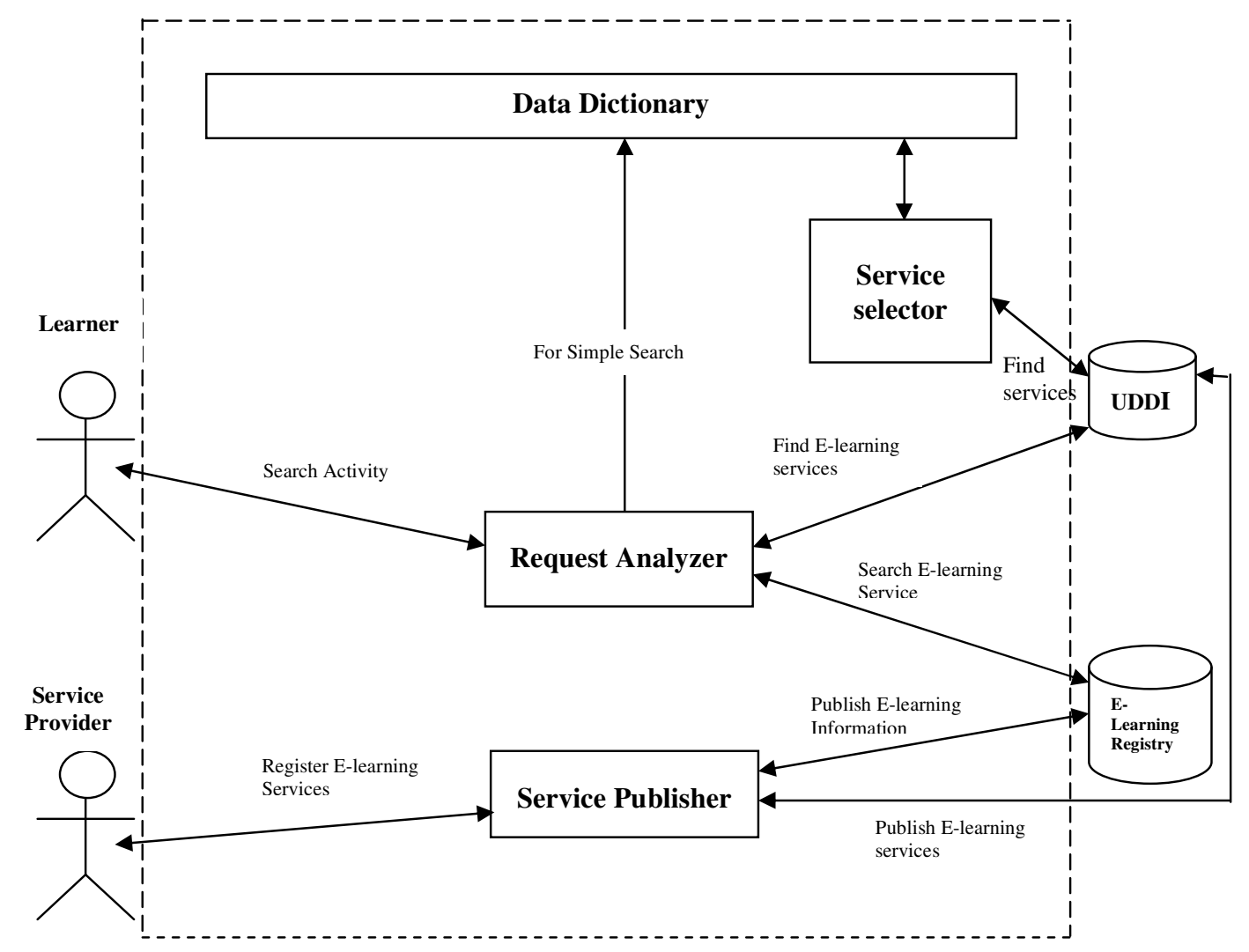

Figure 2. Internal Architecture of E-Learning Broker

\section{E-LEARNING SERVICE DISCOVERY MECHANISM}

In a minimalistic scenario, there exists at least one Web Service provider that publishes some service such as a weather service and a Web Service consumer that uses this service. The Web Service discovery is the process of finding a suitable Web Service for given task of a requester or a composition mechanism. The Service Provider registers its services at the broker and the broker publishes the provider's information into the UDDI. When the Learner searches for the service at the broker, the broker will search for the exact keywords in the database and returns the Object_id for further processing. 


\subsection{The Publish E-Learning Service Mechanism}

The UDDI refers to the Business Entity and stores all the necessary information like the Provider's name and address and its unique ID (key). During publish operation the key is returned to the service provider. Figure 3 shows the sequence of activities involved in publish e-learning service operation. The sequence of activities involved in e-learning service publishing is described below.

- First the E-Learning Service provider should register with the broker, the service it provides.

- The broker then publishes this information in the UDDI and it publishes its description about the learning content in the e-learning registry.

- The broker also sends an acknowledgement to the e-learning service provider that its information has been registered.

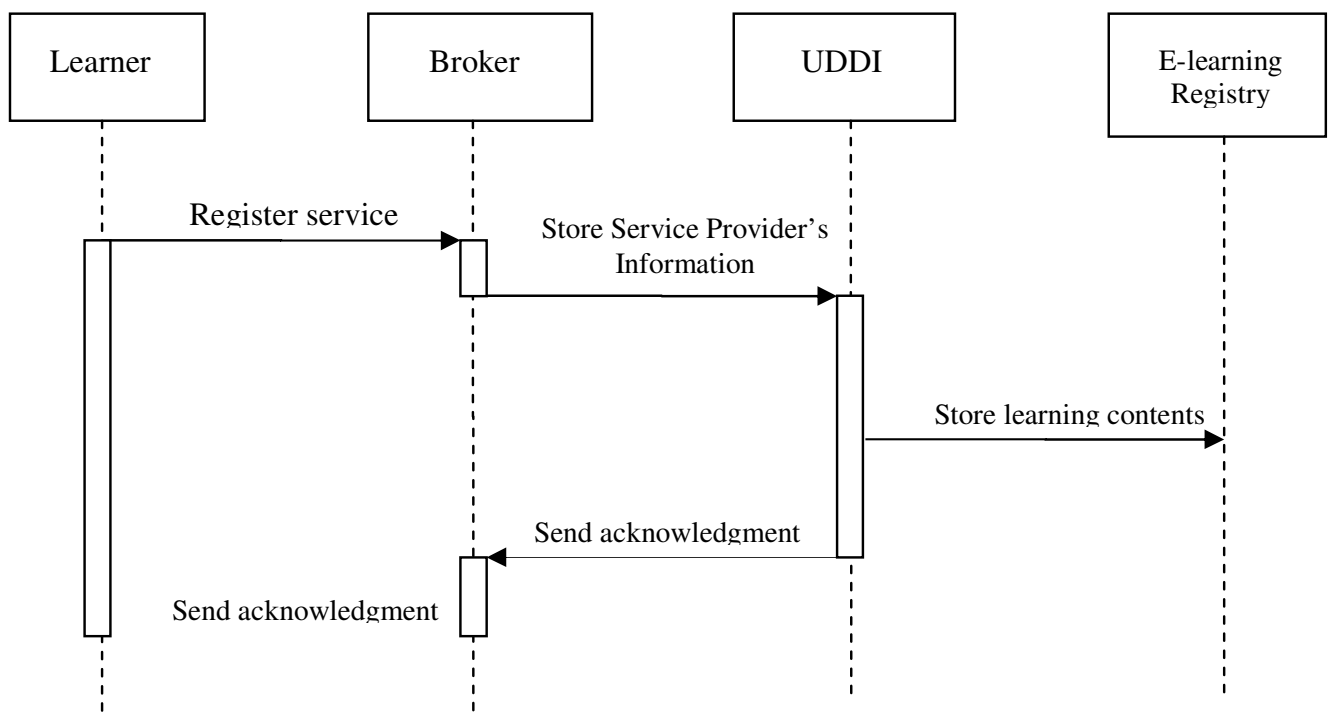

Figure 3. Sequence Diagram for Publishing E-Learning Service Operation

\subsection{The E-Learning Service Discovery (Select) Mechanism}

When the learner searches a learning content by sending the request to the broker in the form of a query, the broker finds the perfect match for the keywords entered by the learner. This is done with the help of data dictionary (WordNet). Using these keywords the appropriate information about the learning content is then retrieved from e-learning registry and then encapsulated in an object. The search result will contain the type of the content (online lecture, videos, articles and textbooks), brief information about the searched content and its URI. Figure 4 depicts the sequence of activities involved in select e-learning service operation.

The sequence of activities involved in e-learning service selection (discovery) is described below.

- The learner sends the query, enriched with functional semantics to the broker for the discovery of relevant e-learning services

- The broker resolves the query and finds the specific keywords to search the learning contents in the e-learning registry as follows:

o The broker finds the perfect match for the query using WordNet and obtains the keywords in XML form

o Using these matched keywords the broker searches the relevant information about the learning material from e-learning registry 
- Once the contents are found, the URIs, object name, object type are encapsulated in an object and returned to the broker

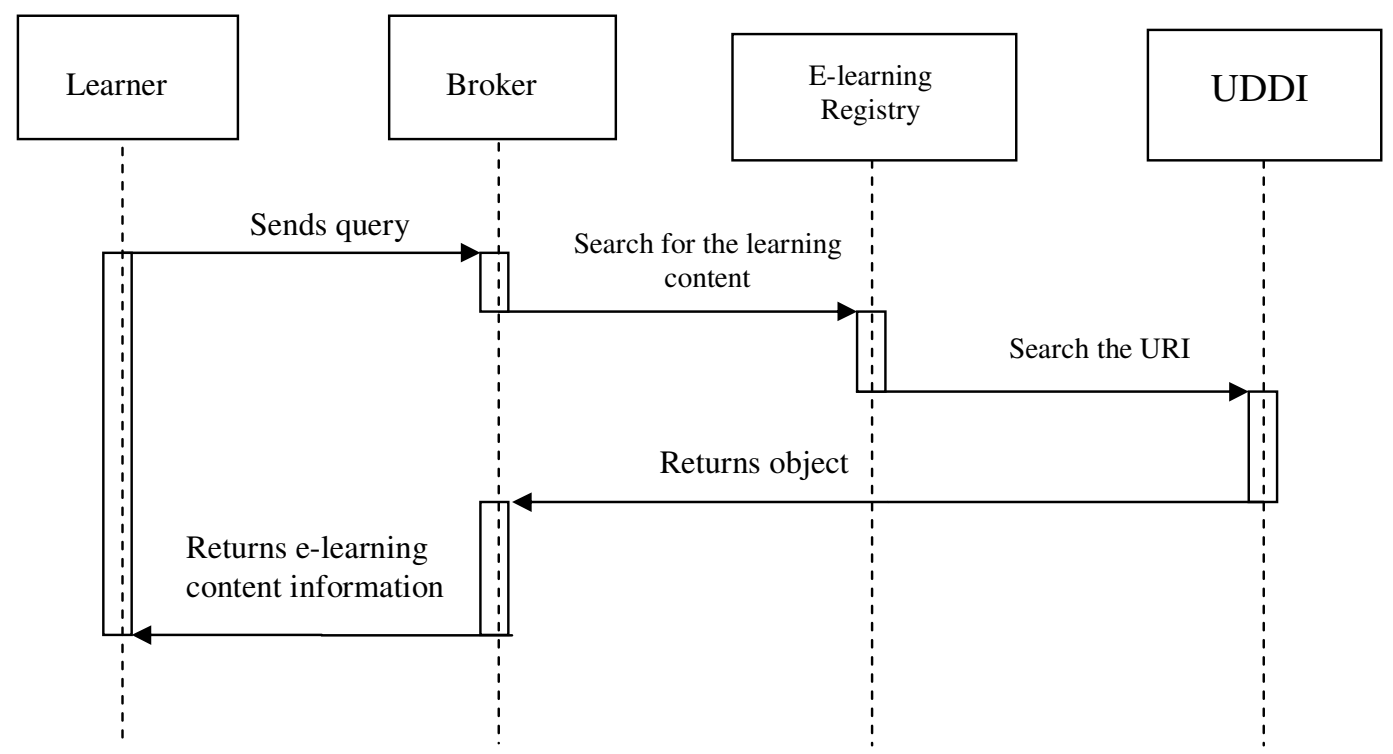

Figure 4. Sequence Diagram for Select E-Learning Service Operation

\section{IMPLEMENTATION AND EXPERIMENTS}

The e-learning service discovery architecture is implemented on Intel Core i5-2430M machine with Microsoft Windows 7 as computing environment. The e-learning service publishing and discovery mechanism is developed using Microsoft Visual Studio 2010 with .NET Framework 4.0. To find the similar words (synonyms) of query the WordNet.Net 2.1 (2005) API is used.

\subsection{Implementation of Publishing and Discovery}

The service provider registers its service through the user interface which calls register_provider_details() function. Figure 5 depicts the user interface created to puchlish the elearning services. Figure 6 presents the user interface designed to search relevant e-learning services. When the learner requests the broker by sending a query, the query is given as a parameter to the readxml() function used by the Data Dictionary. This makes use of WordNet 2.1 to differentiate the exact keywords. The readxml( ) function implicitly calls the WordNet 2.1 using words_match( ) function to find the synonyms and displays the result in an XML format. Using these synonyms the appropriate information about the learning content is then retrieved and encapsulated in an object. This object is returned by the function display_object( ). The search result will contain the type of the content (online lecture, videos, articles, and textbooks), brief information about the searched content and it's URI. This Object is used by the service selector, which separates the contents of this object based on the object type and stores it. The service selector also responsible for finding the perfect binding_template key from the e-learning registry and mapping this key to the binding_template table in the UDDI to retrieve suitable URI based on the order of precedence.

\subsection{Experiments}

Consider the following experiment carried out to verify the effectiveness of the proposed discovery mechanism. The Service provider has a registered service in UDDI for a learning 
material related to the Database Management System and information about various object types of learning materials are available in the e-learning registry.

- The Domain Expert creates an XML file having the various queries and its related synonyms. This is done with the help of WordNet 2.1. This XML file is referred by the broker during the search operation

- The learner fetches for the DBMS related learning materials whose object type is 'text' by sending a query to the broker. If the query sent is "hunt reference book" and subject is entered as "DBMS"

- The broker resolves the query entered by the learner and tokenizes it. The broker uses WordNet 2.1 to find the corresponding synonym of each token using XML form as shown below:

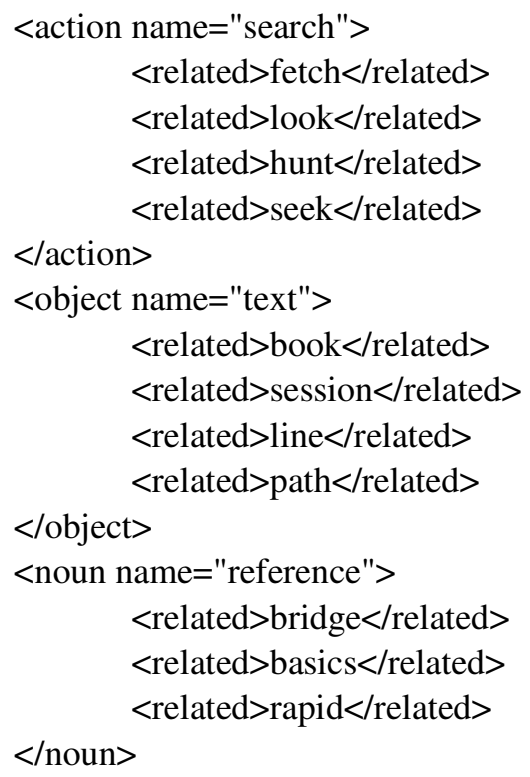

- The WordNet 2.1 differentiates the action, object and noun accordingly and provides it to the e-learning broker

- The output obtained here would be "search reference text". The broker uses these keywords along with the object name, "DBMS" to search for the appropriate learning content in the ELearning Registry

- A sample dataset for the columns in the e-learning registry is as shown in Table 1

Table 1. Tabular Representation of Sample E-learning Registry Contents

\begin{tabular}{|l|l|l|l|l|l|}
\hline Obj_type & Obj_action & Qualifier & Obj name & Obj_id & Bt_key \\
\hline Course & search & Reference & dbms & Obj1 & Btkey1 \\
\hline Session & get & Crash & dbms & Obj2 & Btkey2 \\
\hline Talk & fetch & Rapid & java & Obj3 & Btkey3 \\
\hline
\end{tabular}

- If the match is found then the broker maps this object type to retrieve all necessary information related to DBMS. The content information like type of the content (online lecture, videos, articles, textbooks), a brief information about the searched content, information about the service provider and its URI will be returned to the learner 
- If the match is not found then this query will be written in to an XML file which will be notified to the Domain Expert. The contents of this XML file is shown below: $<$ keywords $>$ hunt reference book DBMS $<$ /keywords $>$

$\leftarrow \rightarrow$ C (Olocalhost:50827/WebSite2/addelearn.aspx

\section{Add E-Learn Information}

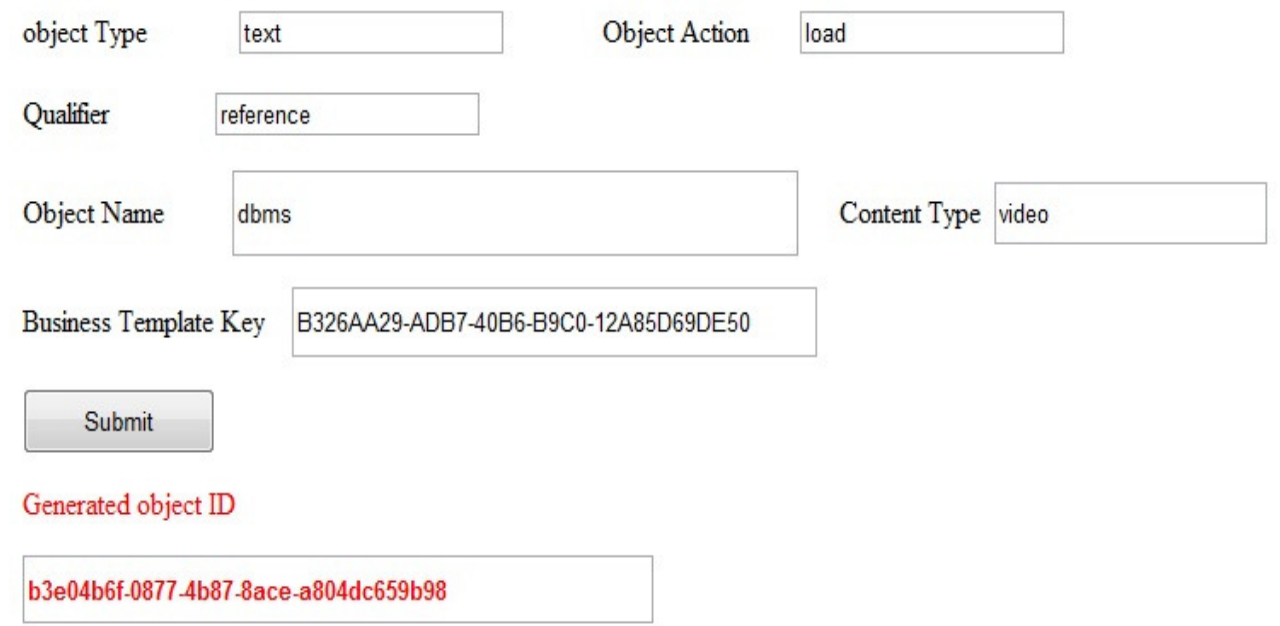

Figure 5. Snapshot of the E-Learning Information Publishing User Interface 


\section{Search Content}

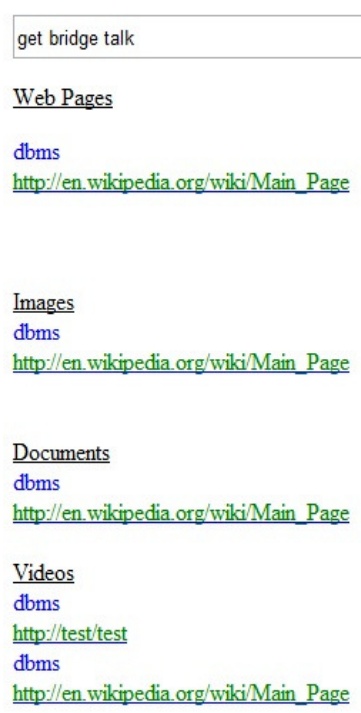

subject dbms

$\underline{\text { Web Pages }}$

$\mathrm{dbms}$

http//en.wikipedia.org/wiki/Main_Page

dbms

Documents

dbms

http://en wikipedia org/wiki/Main_Page

http://en.wikipedia.org/wiki/Main_Page

Figure 6. Snapshot of the E-Learning Service Search User Interface

\section{CONCLUSION}

Web Services provide an attractive alternative for businesses to reach out to their potential customers. This paper addresses the issues related to e-learning service discovery. The quest of learner to find the appropriate learning material can be fulfilled by using this architecture. This research work comprises of the discovery mechanism used to search for the appropriate learning content or digital material. In order to minimize the search only the appropriate synonyms of the service requester's query are obtained from the WordNet which gives better search results. The paper implements broker based architecture which helps in overcoming the disadvantages of traditional e-learning systems. It simplifies the work of searching, categorizing and presentation of learning service information. It also makes it simple for the learner to navigate to the various elearning service providers and thus saves the learners time and effort in searching for the suitable learning contents.

\section{ACKNOWLEDGEMENTS}

This research work is sponsored by "VTU Research Grants Scheme" of Visvesvaraya Technological University, Belgaum, India (No. VTU/Aca./2009-10/A-9/11619 dated: 04/01/2010).

\section{REFERENCES}

[1] Jianhua Jiang, Shuxin Guo and Jie Zhang, "Architecture Design of Campus Information Convergence System for E-learning Based on Web Service Technology", In Proceedings of the 2010, 2nd International Conference on Future Computer and Communication, V2-443, IEEE 2010. 
[2] Afroza Sultana and Ishrat Sultana, "E-School: A Web-Service Oriented Resource Based E-learning System", In Proceedings of the 2010 International Conference on Networking and Information Technology, IEEE 2010.

[3] Gottfried Vossen and Peter Westerkamp, "E-Learning as a Web Service (Extended Abstract)", In Proceedings of the Seventh International Database Engineering and Applications Symposium (IDEAS'03), pp. 242-249, IEEE 2003.

[4] H. Kreger, "Web Services Conceptual Architecture (WSCA.1.0)", Published May 2001, [online] Available from: www.ibm.com/software/ solutions/webservices/pdf/wsca.pdf, [visit: April 2007].

[5] Kerrigan, M (2006). "Web Service Selection mechanisms in the Web Service Execution Environment (WSMX)", In Proceedings of the 21st Annual ACM Symposium on Applied Computing (SAC'06), ACM 1-59593-108-2/06/0004, pp. 1664-1668, ACM.

[6] Xu Wei and Jun Yan "An E-learning System Architecture Based on Web Services and Intelligent Agents", In Proceedings of the International conference on Hybrid Intelligent Systems, 2009. Page(s): $173-177$.

[7] Phankokkruad M and Woraratpanya K, "Web Services for Learning Management Systems: Communication architecture", Communications (MICC), In Proceedings of the IEEE 9th Malaysia International Conference, IEEE 2009.

[8] Santos J.M, Anido L and Llamas M "Design of a Semantic Web-based Brokerage Architecture for the E-learning Domain-A Proposal for a Suitable Ontology”, In Proceedings of the 35th annual conference Frontiers in Education, 2005.

[9] XiaohongQiu, AnumitJooloor "Web Service Architecture for e-Learning", In Proceedings of the Third international conference on Information Technologies, IEEE 2007.

[10] Xiaofei Liu, Abdulmotaleb El Saddik and Nicolas D. Georganas "An Implementable Architecture Of An E-learning System" Advanced Learning Technologies”, In Proceedings of the Fifth IEEE International Conference on Publication, IEEE 2005.

[11] Chtouki, Y Harroud, H Elkhalidi, M Samir "Design and Implementation of E-learning Management System using Service Oriented Architecture", In Proceedings of the International Conference on Information Technology Based Higher Education and Training (ITHET), 2010. Page(s): 219 - 225.

[12] Victor Pankratius, Olivier Sandel and WolffriedStucky, "Retrieving Content with Agents in Web Service E-Learning Systems", $28^{\text {th }}$ annual conference Frontiers in Education, 2003.

[13] Li, B., Tang, X. and LV, J. (2005). "The Research and Implementation of Services Discovery Agent in Web Services Composition Framework.", In Proceedings of the Fourth International Conference on Machine Learning and Cybernetics, Guangzhou, 18-21 August 2005, pp. 78-84, IEEE.

[14] Judith Rodríguez, Luis Anido and Manuel J. Fernández, "How can the Web Services Paradigm improve the E-learning?", In Proceedings of the 3rd IEEE International Conference on Advanced Learning Technologies (ICALT'03), IEEE 2003.

[15] Zhao Gang, Yang ZongKai and Yan Kun, "Design and Implementation of a Distributed Learning Resource Registry System”, In Proceedings of the Fourth International Conference on Computer and Information Technology (CIT'04), IEEE 2004.

[16] Demian Antony D'Mello and Supriya Salian, "Functional Semantics for E-Learning Web Services Description and Discovery", In Proceedings of the Second International Conference on Meta Computing, pp. 109-117, ISBN: 978-81-8424-736-7Goa, India, Dec 2011.

\section{Authors}

Demian Antony D'Mello received his Bachelor degree in Computer Engineering in 1999 from Mangalore University, India and his Master degree in Computer Science and Engineering in 2003 from National Institute of Technology Karnataka (NITK), Surathkal, India. He completed his $\mathrm{PhD}$ from the Department of Information Technology, NITK in the year 2010. Presently, he is working as a professor in the Department of Computer Science and Engineering, St. Joseph Engineering College, Mangalore, India. His research interests are in the areas of Web technologies, Web services, Distributed Computing and Cloud Computing.

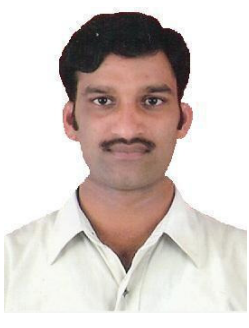

